\title{
Complete axial torsion of the gravid uterus by 180 degrees
}

\author{
Leelavathi Basava*, Priyankur Roy, Sindhu Sree G., Anusha Priya V., Impana S.
}

Department of Obstetrics \& Gynaecology, JSS Medical College \& Hospital, Mysore, India

Received: 19 September 2015

Revised: 03 October 2015

Accepted: 29 October 2015

\author{
*Correspondence: \\ Dr. Leelavathi Basava, \\ E-mail: drleelavathibasava@gmail.com
}

Copyright: (c) the author(s), publisher and licensee Medip Academy. This is an open-access article distributed under the terms of the Creative Commons Attribution Non-Commercial License, which permits unrestricted non-commercial use, distribution, and reproduction in any medium, provided the original work is properly cited.

\begin{abstract}
Minimal rotation of the gravid uterus, till 45 degrees, is a normal finding in the third trimester of pregnancy. However, a pathologic rotation of the uterus beyond 45 degrees-torsion of the entire uterus-is rarely seen in obstetrical practice. We present a case of uterine torsion in pregnancy diagnosed at caesarean section. A 25-years-old primigravida was admitted with pain abdomen and tenderness over the lower abdomen. She underwent emergency LSCS (lower segment caesarean section) for foetal bradycardia. Intra-operatively, the uterus was rotated 180 degrees right to left. There was an urgency to deliver the foetus due to bradycardia and hence, an incision on the posterior wall of the uterus was made as there was no time to delineate the anatomy. Torsion was corrected and the uterus was sutured. Prompt recognition and management of this condition is necessary for better maternal and foetal outcome. Uterine torsion is an infrequently reported and potentially dangerous complication of pregnancy that occurs mainly in the third trimester with adverse maternal and neonatal consequences.
\end{abstract}

Keywords: Pregnancy, Uterus

\section{INTRODUCTION}

Rotation of the gravid uterus is common during pregnancy but rarely exceeds 45 degrees. ${ }^{1}$ It is more common towards the right. The rotation of the uterus on itself, causes decrease in the blood supply, and is called uterine torsion. Uterine venous obstruction causes increased pressure in placental cotyledons leading to abruption and foetal distress; and subsequent progress to uterine artery obstruction can cause reduction in the placental perfusion which can lead to foetal demise. ${ }^{2}$

The aetiological factors could be soft thin-walled lower uterine segment, laxity of the supporting ligaments and abdominal wall, in combination with excessive movements of the foetus, uterine obliquity, or the presence of an asymmetrical uterine fibroid. ${ }^{3}$ Moreover, if in the same circumstances the mother habitually lay on her right side some degree of clockwise axial rotation could possibly occur.

\section{CASE REPORT}

A 25 years old primigravida at term gestation presented to the labour ward of JSS Medical College, Mysore, India with complaints of severe intermittent and colicky abdominal pain of one-day duration. It started acutely but gradually increased in intensity involving whole of the abdomen. On examination, the patient was afebrile. Her vital parameters were stable. The uterus was term size and tender on palpation; foetal heart sound was around $140 \mathrm{bpm}$. On vaginal examination, the cervical os was $2 \mathrm{cms}$ dilated and $50 \%$ effaced.

She was admitted in the labour ward. Admission nonstress test (NST) was reactive. Routine blood and urine investigations were all within normal limits. She ruptured her membranes spontaneously and it was thick meconium stained liquor. There was a sudden foetal bradycardia of $90 \mathrm{bpm}$ noted, and it persisted for almost 5 minutes without any signs of recovery. She was planned for 
emergency caesarean section after valid informed consent.

She underwent caesarean under spinal anaesthesia. Abdomen was opened by pfannenstiel incision. After opening the peritoneum, incision was straight away put on the lower uterine segment as the extraction had to be quick considering the foetal bradycardia. A live female baby of $3 \mathrm{kgs}$ weight was extracted with difficulty. Hind waters were meconium stained. The uterus was exteriorised and then the findings were noted - the ovaries bilaterally were the anterior most structures followed by the broad and the round ligament. On manipulation, uterine torsion of 180 degrees was noted and the incision made was on the posterior surface of the uterus (Figure 1).

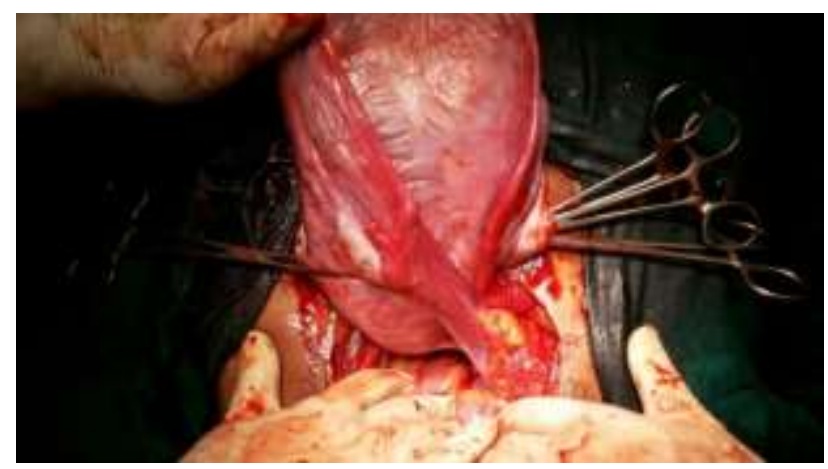

Figure 1: Torsion of the uterus seen intra-operatively.

Uterine detorsion was performed. After correcting the torsion, the thick posterior wall incision was noted (Figure 2). The incision site was sutured in 2 layers using Vicyrl No. 1 (Figure 3) and put back into abdomen. Abdomen was closed. She recovered well and was discharged on $7^{\text {th }}$ post-operative day.

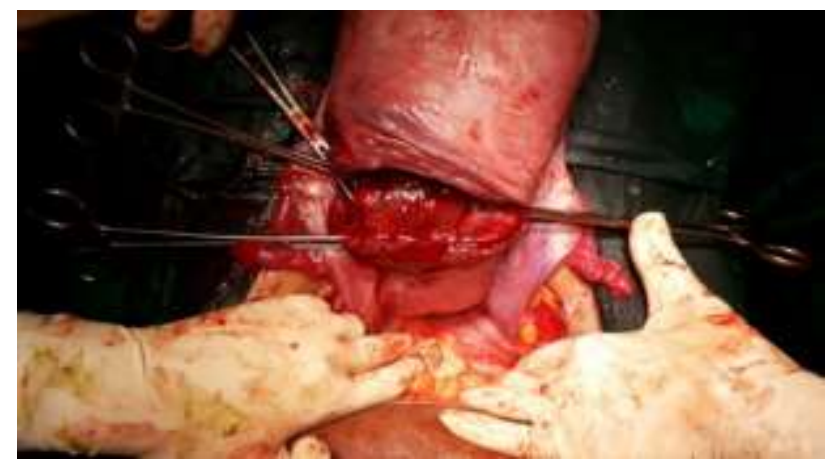

Figure 2: Intra-operative photograph of the uterus showing incision on the posterior wall.

\section{DISCUSSION}

Uterine torsion is defined as rotation of the uterus of more than 45 degrees on its long axis. It can range from 60-720 degrees. There is dextrorotation in two-third and levorotation in one-third of cases. The exact etiology is not known. According to Wilson et al. ${ }^{3}$ most of cases had normal anatomy that is unexplained torsion, whereas according to Piot et al $31.8 \%$ had uterine leiomyoma, $14.9 \%$ uterine anomalies especially bicornuate uterus, $8.4 \%$ had pelvic adhesions, $7 \%$ had ovarian cysts, $4.6 \%$ had abnormal presentation and foetal anomalies, $2.8 \%$ abnormalities of spine and pelvis and the rest did not have any discoverable cause. ${ }^{1}$ Some other causes of uterine torsion have also been reported. Salani et al reported a case where ECV (external cephalic version) caused uterine torsion. ${ }^{4}$ They recommended to add torsion as one of complications of ECV. Duplantier et al reported a case of torsion due to maternal trauma. ${ }^{5}$ Achanna et al have reported a case of torsion in uterus didelphys due to abdominal massage during labour by traditional birth attendants. ${ }^{6}$

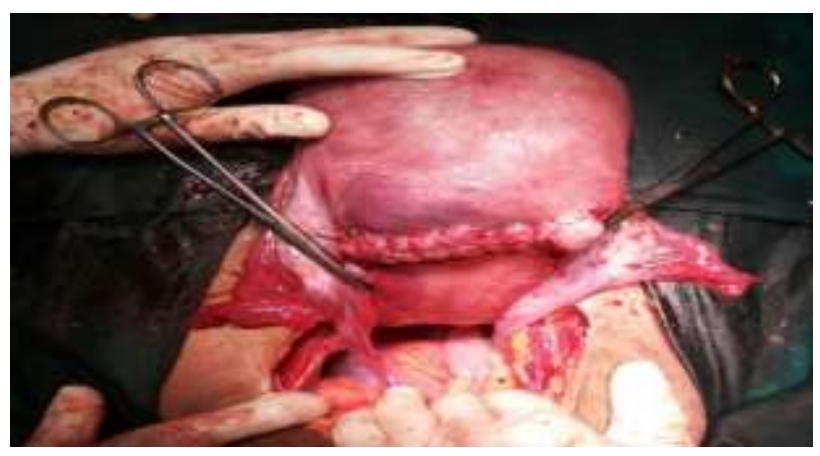

Figure 3: Intra-operative photograph showing posterior uterine wall incision which has been sutured in 2 layers and haemostasis has been achieved.

Jensen suggested that uterine torsion can occur in all age groups, all parity, and all stages of pregnancy. ${ }^{2}$ Most cases are similar to our case with abdominal pain and tenderness and diagnosed only at caesarean section. They may present with birth obstruction, vaginal bleeding, shock, urinary and intestinal symptoms. High degree of suspicion is needed to diagnose this condition antenatally. Gule et al used modification of placental site compared to previous scan on USG (ultrasonography) and abnormal position of ovarian vessels across uterus on doppler to diagnose torsion. $^{7}$

Nicholson et al suggested X-shaped configuration of upper vagina on MRI (magnetic resonance imaging) as a sign to diagnose torsion. ${ }^{8}$ This is based upon the fact that vagina is normally seen on MRI as an $\mathrm{H}$-shaped structure, but with torsion of the uterus and upper vagina, the vagina appears as an $\mathrm{X}$-shaped structure. Management requires emergency laparotomy. At term, uterus is derotated and LSCS (lower segment cesarean section) is done. If derotation is not possible, posterior low transverse incision is made and the foetus is extracted as it was done in our case. ${ }^{9}$ In such cases elective section is advised in next pregnancy as risk of rupture is very high. ${ }^{3}$

There are no reported cases of maternal deaths in the literature. Delineation of proper anatomy is necessary to 
prevent injury to major vessels and organs. ${ }^{10}$ In mid trimester, if the patient is symptomatic and uterine torsion is diagnosed, uterus can be derotated and pelvic pathology causing torsion removed like myomectomy and ovarian cystectomy. To prevent recurrent torsion some have advocated plication of round ligament. Mustafa et al have reported a case of plication of uterosacral ligament to prevent recurrent torsion. ${ }^{11}$

\section{CONCLUSIONS}

The usual clinical presentation of torsion of the gravid uterus is vague, by knowing the risk factors we can suspect the condition and confirm it by MRI. This will lead to the better management of the condition with good maternal and foetal outcome.

Funding: No funding sources Conflict of interest: None declared

Ethical approval: Not required

\section{REFERENCES}

1. Piot D, Gluck M, Oxorn H. Torsion of gravid uterus. The Canadian Medical Association Journal. 1973;109(10):1010-1.

2. Jensen JG. Uterine torsion in pregnancy. Acta Obstetricia et Gynecologica Scandinavica. 1992;71:260-5.

3. Wilson D, Mahalingham A, Ross S. Third trimester uterine torsion: A case report. Journal of Obstetrics and Gynaecology Canada. 2006;28(6):531-5.

4. Salani R, Theiler RN, Lindsay M. Uterine torsion and foetal bradycardia associated with external cephalic version. Obstet and Gynaecol. 2006;108(3):820-2.
5. Duplantier N, Begneaud W, Wood R, Dabezies C. Torsion of a gravid uterus associated with maternal trauma: A case report. Journal of Reproductive Medicine for Obstetrician and Gynaecologist. 2002;47(8):683-5.

6. Achanna S, Monga D, Suhaimi Hassan M. Case report: Torsion of a gravid horn of didelphic uterus. Journal of Obstetrics \& Gynaecology Research. 1996;22(2):107-9.

7. Gule P, Adjobi R, Nguessan E. Uterine torsion with maternal death: Our experience and literature review. Clinical and Experimental Obstetrics \& Gynaecology. 2005;32(4):245-6.

8. Nicholson WK, Coulson CC, McCoy MC, Semelka RC. Pelvic magnetic resonance imaging in the evaluation of uterine torsion. Obstet and Gynaecol. 1995;85(5):888-90.

9. Albayrak M, Berian A, Ozdenir I, Deniraran Y, Guralp O. Deliberate posterior low transverse incision at caesarean section of gravid uterus in $180^{\circ}$ of torsion: A case report. Journal of Reproductive Medicine. 2011:56:181-3.

10. Bolaji II, Rafla NM, Mylotte MJ. Classical caesarean section through the posterior uterine wall. Irish Journal of Medical Science. 1992;161(2):46-7.

11. Mustafa MS, Shakeel F, Sporrong B. Extreme torsion of the pregnant uterus. Australia and New Zealand Journal of Obstetrics \& Gynaecology. 1999;39(3):360-3.

Cite this article as: Basava L, Roy P, Sindhu Sree G, Anusha Priya V, Impana S. Complete axial torsion of the gravid uterus by 180 degrees. Int $\mathrm{J}$ Reprod Contracept Obstet Gynecol 2015;4:2074-6. 